\title{
Automating UbiFast for High-throughput and Multiplexed Ubiquitin Enrichment
}

\author{
Keith D. Rivera ${ }^{1}$, Meagan E. Olive ${ }^{1}$, Erik J. Bergstrom¹, Alissa J. Nelson², Kimberly A. Lee², \\ Shankha Satpathy ${ }^{1}$, Steven A. Carr ${ }^{1 \otimes}$ \& Namrata D. Udeshi ${ }^{1, \square}$
}

${ }^{1}$ Broad Institute of MIT and Harvard, Cambridge, MA 02142, USA.

${ }^{2}$ Cell Signaling Technology, Danvers, MA 01923, USA

corresponding authors: udeshi@broadinstitute.org, scarr@broad.mit.edu

RUNNING TITLE: Automating UbiFast for Multiplexed Ubiquitin Enrichment

\begin{abstract}
Robust methods for deep-scale enrichment and site-specific identification of ubiquitylation sites is necessary for characterizing the myriad roles of protein ubiquitylation. To this end we previously developed UbiFast, a sensitive method for highly multiplexed ubiquitylation profiling where K-E-GG peptides are enriched with antiK- $\varepsilon-G G$ antibody and labeled on-antibody with isobaric labeling reagents for sample multiplexing. Here, we present robotic automation of the UbiFast method using a magnetic bead-conjugated K- $\varepsilon-G G$ antibody (mK- $\varepsilon-G G$ ) and a magnetic particle processor. We report the identification of $\sim 20,000$ ubiquitylation sites from a TMT10plex with $500 \mu \mathrm{g}$ input per sample processed in $\sim 2$ hours. Automation of the UbiFast
\end{abstract}


Automating UbiFast for Multiplexed Ubiquitin Enrichment

method greatly increased the number of identified and quantified ubiquitylation sites, improved reproducibility and significantly reduced processing time. The workflow enables processing of up to 96 samples in a single day making it suitable to study ubiquitylation in large sample sets. Here we demonstrate the applicability of this method to profile small amounts of tissue using breast cancer patient-derived xenograft (PDX) tissue samples.

\section{Introduction}

Ubiquitylation is a highly-conserved protein post-translational modification regulating a wide variety of cellular functions including regulation of protein turnover through the ubiquitin-proteasome system ${ }^{1}$. The ubiquitylation process is regulated by E1 activating, E2 conjugating, E3 ligating enzymes together with deubiquitinases.

Dysregulation of ubiquitylation enzymes and deubiquitinases can lead to aberrant activation or deactivation of pathways involved in many disease processes, notably cancer progression, neurodegeneration and innate and adaptive immune regulation ${ }^{2}$. Drugs targeting the ubiquitin system such as proteasome inhibitors have proven highly successful in the clinic. The development of additional therapeutics targeting this pathway are emerging but remain highly dependent on continued understanding of ubiquitination biology in disease ${ }^{3-5}$.

Liquid chromatography-mass spectrometry (LC-MS/MS) is the leading method for unbiased analysis of protein modifications, including protein ubiquitylation ${ }^{6,7}$.

Analysis of ubiquitylated proteins is typically carried out by first using trypsin to generate peptides suitable for LC-MS/MS analysis. Trypsin cleaves proteins at the carboxyl side 
Automating UbiFast for Multiplexed Ubiquitin Enrichment

of lysine (Lys) and arginine (Arg). When ubiquitin is attached to a substrate protein, trypsin digestion leaves a glycine-glycine (GG) remnant on the side chain of Lys residues of tryptic peptides which were formerly ubiquitylated. Antibodies that recognize this di-glycl remnant (K-ع-GG) are used to enrich ubiquitylated (Ub) peptides for analysis by liquid-chromatography- tandem mass spectrometry (LC-MS/MS) ${ }^{8-11}$.

Isobaric chemical tags such as the Tandem Mass Tag (TMT) system are commonly used to compare up to 16 samples within a single experiment and provide precise relative quantitation of peptides and proteins ${ }^{12-14}$. A major limitation of integrating TMT quantitation and ubiquitylation profiling with $\mathrm{K}-\varepsilon-\mathrm{GG}$ antibodies is that these antibodies no longer recognize and enrich peptides when the $\mathrm{N}$-terminus of the di-glycyl remnant is derivatized with TMT. To overcome this limitation, we recently developed the UbiFast method for highly sensitive and multiplexed analysis of ubiquitylation sites from cells or tissue ${ }^{15}$. The UbiFast method employs an anti-K-E-GG antibody for enrichment of K-ع-GG peptides followed by on-antibody TMT labeling. Specifically, K-ع-GG peptides are labeled with TMT reagents while still bound to the anti-K- $\varepsilon-G G$ antibody which allows the NHS-ester group of the TMT reagent to react with the peptide $\mathrm{N}$-terminal amine group and the $\varepsilon$-amine groups of lysine residues, but not the primary amine of the di-glycyl remnant. In this way, TMT-labeled K-ع-GG peptides from each sample are combined, eluted from the antibody, and analyzed by LC-MS/MS. The UbiFast approach eliminates cell culture restrictions,greatly reducing the amount of input material required when using SILAC-based experiments ${ }^{8,9,16-22}$ and improves Ub-peptide recovery and analysis time compared to in-solution TMT labeling 
Automating UbiFast for Multiplexed Ubiquitin Enrichment

strategies $^{23}$. The sensitivity of the UbiFast method makes it suitable for studies in primary tissue samples.

Although the UbiFast method is highly effective for deep-scale LC-MS/MS analysis of ubiquitylated peptides, the throughput is limited because all workflow steps are manually executed, making the procedure laborious. Additionally, an initial crosslinking step is necessary to covalently couple the antibody to agarose beads, which prevents contamination of antibody in enriched samples ${ }^{9}$. Further, slight variations during the K-ع-GG peptide enrichment step can result in an increased variability across replicates within a given TMT experiment.

To improve the UbiFast method, here we evaluate and optimize use of a commercially available anti-K- $\varepsilon-G G$ antibody supplied irreversibly coupled to magnetic

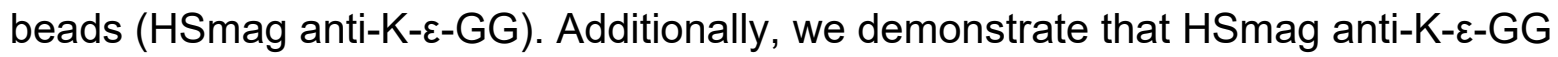
antibody enables automation of the UbiFast method on a magnetic bead processor greatly increasing sample processing throughput while maintaining low variation across experimental replicates (Figure 1). We also show that the automated UbiFast method can be easily scaled to process multiple 10-plex TMT experiments in just a few hours. The automated UbiFast method was benchmarked against the previously published, manually executed UbiFast method for profiling patient-derived breast cancer xenograft tissue.

\section{Experimental Procedures}

\section{In-solution digestion of Jurkat cell lysates}

Jurkat cells were grown in suspension with RPMI 1640 medium, glutaMAX supplement (Life Technologies) and 10\% heat-inactivated fetal bovine serum (Life 
Automating UbiFast for Multiplexed Ubiquitin Enrichment

Technologies). Cells were pelleted, washed $2 x$ with 1 X PBS ( $\mathrm{pH} \mathrm{7.4),} \mathrm{and} \mathrm{lysed.} \mathrm{Lysis}$ buffer consisted of $8 \mathrm{M}$ urea, $50 \mathrm{mM}$ tris hydrochloride (Tris-HCl) pH 8.0, $150 \mathrm{mM}$ sodium chloride $(\mathrm{NaCl})$ and $1 \mathrm{mM}$ ethylenediaminetetraacetic acid (EDTA). Immediately before lysing the following additives were added to their respective final concentrations: aprotinin (Sigma) to $2 \mathrm{ug} / \mathrm{mL}$, leupeptin (Roche) to $10 \mathrm{ug} / \mathrm{mL}$, phenylmethylsulfonyl fluoride (PMSF) (Sigma) to $1 \mathrm{mM}, \mathrm{PR}-619$ (LifeSensors) to $50 \mathrm{uM}$ and chloroacetamide (CAA) to $1 \mathrm{mM}$. Cells were lysed for $30 \mathrm{~min}$ on ice and then centrifuged for $10 \mathrm{~min}$ at $20,000 \times \mathrm{g}$ and $4^{\circ} \mathrm{C}$. Protein concentration was then determined using a bicinchoninic acid (BCA) assay kit (ThermoFisher Scientific). The lysate was diluted to $8 \mathrm{mg} / \mathrm{mL}$ with lysis buffer, dithiothreitol (DTT) was added to a final concentration of $5 \mathrm{mM}$ and it was incubated at room temperature (RT) for $45 \mathrm{~min}$. Next, iodoacetamide (IAA) was added to a final concentration of $10 \mathrm{mM}$ and the lysate was incubated for 30 minutes at RT in the dark. Then the lysate was diluted 1:4 with $50 \mathrm{mM}$ Tris-HCL, pH 8.0. After dilution Lys-C (Wako) was added with an enzyme to substrate ratio of 1:50 and the lysate was incubated for $2 \mathrm{hrs}$ at RT. Trypsin was then added with an enzyme to substrate 1:50 and the lysate was incubated overnight at RT. The following morning neat formic acid (FA) was added to a final concentration of $1 \%$ and the sample was centrifuged for 10 min at $10,000 \times g$ to remove urea and undigested proteins. Peptides were cleaned up using a $500 \mathrm{mg}$ Sep-Pak tC18 solid phase extraction cartridge (Waters). Briefly, the cartridge was conditioned with $5 \mathrm{~mL}$ of Acetonitrile (ACN) / 0.1\% FA and then $50 \% \mathrm{ACN}$ / $0.1 \%$ FA. Equilibration was performed with 4 additions of $5 \mathrm{~mL} 0.1 \%$ trifluoroacetic acid (TFA). Digested peptides were loaded and then washed twice with $5 \mathrm{~mL} 0.1 \%$ TFA. Peptides were eluted with 2 additions of $50 \%$ ACN / 0.1\% FA. The eluate was frozen at 
Automating UbiFast for Multiplexed Ubiquitin Enrichment

$-80^{\circ} \mathrm{C}$ and dried to completion in vacuo. Peptides were reconstituted in $30 \% \mathrm{ACN} / 0.1 \%$

FA and peptide concentration was measured using a BCA assay kit. The peptides were divided into $500 \mu \mathrm{g}$ aliquots, frozen at $-80^{\circ} \mathrm{C}$ and dried in vacuum. Once dried the peptide aliquots were stored at $-80^{\circ} \mathrm{C}$ until use.

\section{Label-free comparison of manual immunoprecipitation with agarose vs. magnetic} beads

Agarose beads used in all antibody comparisons were from the PTMScan ${ }^{\circledR}$ Ubiquitin Remnant Motif (K-ع-GG) Kit (Cell Signaling Technology, Kit \#5562). Preparation of agarose beads included cross-linking as described previously ${ }^{15}$. All agarose bead washes were performed with immunoprecipitation (IAP) buffer (50 mM MOPS, pH 7.2, $10 \mathrm{mM}$ sodium phosphate, $50 \mathrm{mM} \mathrm{NaCl}$ ). Magnetic beads for all experiments come from PTMScan® HS Ubiquitin/SUMO Remnant Motif (K-ع-GG) Kit (Cell Signaling Technology, Kit \#59322), along with 1X HS IAP Bind Buffer \#1 and 1X HS IAP Wash Buffer. Magnetic beads were used as provided, with no additional crosslinking. Input for manual comparisons was $500 \mu \mathrm{g}$ digested Jurkat peptide, and enrichments were performed in triplicate.

Jurkat samples were reconstituted with $1.5 \mathrm{ml}$ of either IAP buffer for agarosebead IPs or 1X HS IAP Bind Buffer \#1 for magnetic-bead IPs. Reconstituted peptides were confirmed to be $\mathrm{pH} 7$ and were spun at $10,000 \times \mathrm{g}$ for $5 \mathrm{~min}(\mathrm{pH}$ adjusted with $1 \mathrm{M}$ Tris if necessary). After cross-linking, agarose beads were suspended in a slurry of 1:25 beads to IAP buffer from which $62.5 \mu$ l slurry (31.25 $\mu$ g antibody) was used per IP. 
Automating UbiFast for Multiplexed Ubiquitin Enrichment

Magnetic beads are provided in a $20 \%$ slurry, and enough was removed for a given experiment to be washed $3 \mathrm{x}$ with $1 \mathrm{ml}$ ice-cold $1 \mathrm{X}$ PBS, inverted $\sim 5 \mathrm{x}$ with each wash, and resuspended again in original volume. Different magnetic bead amounts were tested for this label-free comparison, including $10 \mu$ l slurry $(2 \mu$ l beads) and $5 \mu$ l slurry ( 1 $\mu$ l beads) per IP. Beads were aliquoted into clean $1.5 \mathrm{ml}$ snap-cap Eppendorf tubes.

Reconstituted peptides were added to aliquoted antibody beads and incubated at $4{ }^{\circ} \mathrm{C}$ for $1 \mathrm{~h}$, gently rotating end-over-end. Buffers were chilled during this enrichment step, and all remaining wash steps were performed on ice when tubes were not being handled. After incubation, agarose beads were centrifuged at 2,000 $\mathrm{xg}$ for $1 \mathrm{~min}$ and allowed to settle for $\sim 10 \mathrm{~s}$ before removing supernatant as IP flowthrough.

Simultaneously, magnetic beads were spun at 2,000 x g for $5 \mathrm{~s}$ and allowed to settle for 10 s on a magnetic rack before slowly removing flowthrough. Sample flow throughs can be stored at $-80^{\circ} \mathrm{C}$ for serial analyses.

All washes were performed as quickly as possible. For agarose bead IPs, beads were washed $4 \mathrm{x}$ with $1.5 \mathrm{ml}$ PBS, inverting $\sim 5 \mathrm{x}$ with each wash, centrifuging at 2,000 $\mathrm{x}$ $\mathrm{g}$ for $1 \mathrm{~min}$, letting beads settle and aspirating supernatant. Magnetic beads were washed $4 \mathrm{x}$ with $1 \mathrm{ml} 1 \mathrm{X}$ HS IAP Wash Buffer and 2x with $1 \mathrm{ml} \mathrm{HPLC} \mathrm{H}_{2} \mathrm{O}$, inverting $\sim 5 x$ with each wash, centrifuging for $\sim 5 \mathrm{~s}$ at $2,000 \times \mathrm{g}$, and allowing beads to be drawn to magnet for $\sim 10 \mathrm{~s}$ before aspirating supernatant. Ubiquitin peptides were eluted from all beads with $50 \mu \mathrm{l} 0.15 \%$ TFA for 10 min at RT, gently flicking the beads into solution every 2-3 min. While eluting, 2-punch Empore C18 (3M) StageTips were conditioned and equilibrated $1 \mathrm{x}$ with $100 \mu \mathrm{l}$ methanol (MeOH), $1 \mathrm{x}$ with $100 \mu \mathrm{l} 50 \%$ ACN/0.1\% FA, and $2 x$ with $1 \%$ FA. 
Automating UbiFast for Multiplexed Ubiquitin Enrichment

Upon completion of the first elution, samples were centrifuged quickly at 2,000 $\mathrm{x}$ $\mathrm{g}$, placed on a magnetic rack where applicable, and the supernatant containing ubiquitin peptides was loaded onto its designated conditioned StageTip. The elution step was repeated and the supernatant similarly loaded onto the StageTip to be washed $2 x$ with $100 \mu \mathrm{l} 1 \% \mathrm{FA}$ and eluted with $50 \mu \mathrm{l} 50 \%$ ACN/0.1\% FA. Desalted eluates were transferred to HPLC vials, frozen at $-80^{\circ} \mathrm{C}$, and dried via vacuum centrifugation.

Samples were reconstituted in $9 \mu \mathrm{l} 3 \% \mathrm{ACN} / 0.1 \% \mathrm{FA}$ and $4 \mu \mathrm{l}$ was analyzed via nanoflow liquid chromatography coupled to tandem mass spectrometry, or LC-MS/MS, using an Easy-nLC 1200 system (Thermo Fisher Scientific) online with a Q-Exactive HF-X mass spectrometer (Thermo Fisher Scientific). Samples were injected and chromatographically separated on a fused silica microcapillary column (360 $\mu \mathrm{m}$ OD X $75 \mu \mathrm{m}$ ID) with a $10 \mu \mathrm{m}$ electrospray emitter tip (New Objective) packed to $\sim 25 \mathrm{~cm}$ with ReproSil-Pur $1.9 \mu \mathrm{m} \mathrm{C18-AQ} \mathrm{beads} \mathrm{(Dr.} \mathrm{Maisch} \mathrm{GmbH}$ ) and heated to $50^{\circ} \mathrm{C}$. Online separation occurred over a 154 min gradient, employing a changing ratio of solvent $\mathrm{A}$ (3\% ACN/0.1\% FA) to solvent B (90\% ACN/0.1\% FA). Gradient steps as min:\% solvent B include $0: 2,2: 6,122: 35,130: 60,133: 90,143: 90,144: 50,154: 50$, starting at a mobile phase flow rate of $200 \mathrm{nl} / \mathrm{min}$ for the first six steps and increasing to $500 \mathrm{nl} / \mathrm{min}$ for the final two.

Ion acquisition was performed with a data-dependent analysis method. MS1 scans were collected across a range of $300-1800 \mathrm{~m} / \mathrm{z}$ at a resolution of 60,000 , with an automatic gain control (AGC) target of 3E6 ions and maximum injection time of $10 \mathrm{~ms}$. Within a scan, the top 20 most abundant peaks were picked for higher-energy collisional dissociation (HCD) fragmentation using a collision energy of 28 and isolation window of 
Automating UbiFast for Multiplexed Ubiquitin Enrichment

$0.7 \mathrm{~m} / \mathrm{z}$. MS2 spectra were acquired in centroid mode at a resolution of 45,000 with an AGC target of $1 E 5$ and maximum injection time of $150 \mathrm{~ms}$. Peptide matching was set to "preferred", dynamic exclusion was $20 \mathrm{~s}$, and ions with unassigned charge or charge $=1$ or $>7$ were excluded.

\section{Comparison of TMT labeling efficiency with agarose vs. magnetic beads}

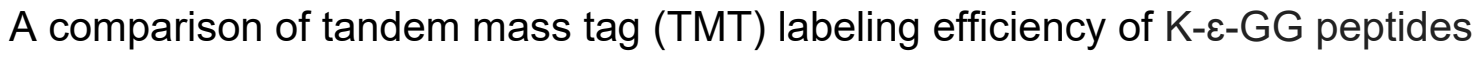
captured by agarose versus magnetic antibody beads was done in triplicate, using 500 $\mu \mathrm{g}$ Jurkat peptide input. Beads were aliquoted as described above, using $62.5 \mu \mathrm{l}$ agarose slurry and $5 \mu \mathrm{l}$ magnetic slurry per IP. Sample reconstitution, incubation with antibody, and flowthrough collection were performed exactly as outlined above. Samples enriched with agarose or magnetic beads were washed once with $1 \mathrm{ml}$ IAP buffer or 1 X HS IAP Wash buffer and once with $1 \mathrm{ml}$ 1X PBS or HPLC $\mathrm{H}_{2} \mathrm{O}$, respectively. With each wash, samples were inverted $\sim 5$ times, centrifuged at 2,000 x g for $\sim 5$ s and allowed to settle on ice or a magnetic rack before aspirating the supernatant.

After washing and immediately before labeling, all beads were resuspended in 200 l 100 mM HEPES (pH 8.5). Each sample was labeled with a single TMT channel (126C, 127N, 127C for agarose replicates and 128N, 128C, 129N for magnetic replicates), adding $400 \mu \mathrm{g}$ reagent in $10 \mu \mathrm{l}$ anhydrous ACN directly to the resuspended beads. Tubes were shaken at $1400 \mathrm{rpm}$ for $10 \mathrm{~min}$ at RT. Labeling was then quenched with $8 \mu \mathrm{l} 5 \%$ hydroxylamine followed by 5 additional minutes of shaking at $1400 \mathrm{rpm}$ at RT. Excess reagent was washed away $1 x$ with $1.3 \mathrm{ml}$ and $2 x$ with $1.5 \mathrm{ml}$ respective IAP 
Automating UbiFast for Multiplexed Ubiquitin Enrichment

wash buffers, inverting tubes $\sim 5$ times, centrifuging for $\sim 5$ at 2,000 $x \mathrm{~g}$ and allowing beads to settle before removing the supernatant. Beads were similarly washed $2 x$ with $1.5 \mathrm{ml}$ 1X PBS (agarose) or HPLC H${ }_{2} \mathrm{O}$ (magnetic) before eluting and stage tipping exactly as described above.

Samples were analyzed similarly to the previous label-free experiment, with the reconstitution and liquid chromatography method being identical. Data acquisition was performed using an Orbitrap Exploris 480 mass spectrometer (Thermo Fisher Scientific) with a data dependent analysis method. MS1 parameters were the same as above, with the exception of a $100 \%$ normalized AGC target. A precursor fit filter was employed, with a fit threshold of $50 \%$ and window of $1.4 \mathrm{~m} / \mathrm{z}$. MS2 spectra were collected with an HCD collision energy of $32 \%$, resolution of 15,000 , AGC target of $50 \%$, and maximum injection time of $120 \mathrm{~ms}$. Remaining ion acquisition parameters were the same as previously described.

\section{Developing Enrichment of K-E-GG peptides using the KingFisher Flex}

For all optimization enrichments $500 \mu \mathrm{g}$ of dried Jurkat peptides were reconstituted in $250 \mu$ I PTMScan ${ }^{\circledR}$ HS IAP Bind Buffer \#1 with a final concentration of 0.01\% CHAPS and placed in a sonicator bath for 2 minutes. $\mathrm{pH}$ was confirmed to be neutral and then solution was cleared by centrifugation for 5 minutes at $10,000 \mathrm{x}$. Magnetic beads from PTMScan® HS Ubiquitin/SUMO Remnant Motif (K-ع-GG) Kit were prepared as described above. After washing beads $3 x$ with ice cold 1x PBS beads were reconstituted to their original volume with HS IAP Bind Buffer \#1 and beads were aliquoted into individual wells of a 96 well KingFisher plate $200 \mu$ l (ThermoFisher 
Automating UbiFast for Multiplexed Ubiquitin Enrichment

Scientific). The solution of cleared peptides was added to the wells containing beads, the plate was covered with aluminum sealing film (Axygen) and rotated end-over-end for 1 hour at $4^{\circ} \mathrm{C}$, except where otherwise noted.

In order to determine if CHAPS is a necessary addition to buffers when using the KF we processed triplicate ubiquitin enrichments with all buffers containing either $0.01 \%$ CHAPS or all buffers without CHAPS added. To assess the feasibility of performing the $\mathrm{K}-\varepsilon-\mathrm{GG}$ peptide capture step on the KF, enrichments were done in triplicate on the KingFisher for 1 hour at room temperature with medium mixing. Separately, duplicate enrichments were done in 96-well KingFisher plates offline with end-over-end rotating in a cold room at $4^{\circ} \mathrm{C}$. The wash buffer used for these experiments was HS IAP Wash Buffer with a final concentration of $0.01 \%$ CHAPS.

To test the viability of using lower volumes of beads, $5 \mathrm{uL}, 10 \mu \mathrm{l}$ and $20 \mu \mathrm{l}$ of slurry were processed in quintuplicate using the label-free KingFisher Flex method described below. Each replicate was injected separately onto the mass spectrometer.

In the interest of assessing the optimal wash buffer, HS IAP Wash Buffer with $0.01 \%$ CHAPS was compared with a modified version of this buffer where it was diluted 1:1 with ACN to make $50 \%$ ACN, $50 \%$ HS IAP Wash Buffer with $.005 \%$ CHAPS.

For label-free experiments a 6-step KingFisher Flex method was used. Step 1 collects the beads from the incubation plate containing the jurkat peptides and the magnetic beads. For comparisons where incubation of peptides and beads is performed on the KingFisher Flex this step mixes the plate at medium speed for 1 hour.

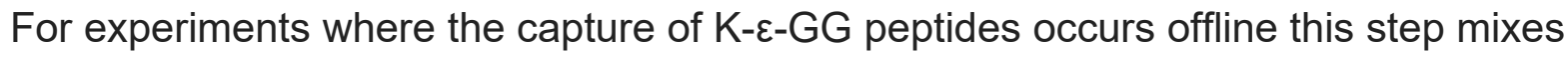
the plate at medium speed for 30 seconds and then collects the beads. Steps 2 through 
Automating UbiFast for Multiplexed Ubiquitin Enrichment

4 are 1 minute washes with $250 \mu$ l of washing buffer where the first 15 seconds are on the bottom mix setting and the remaining 45 seconds are set to fast mixing. Step 5 is a wash for 1 minute with $250 \mu$ l of HPLC water with medium mixing. The final step is elution in $100 \mu \mathrm{l}$ of $0.15 \%$ TFA for 10 minutes with slow mixing. For all steps a collect count of 5 and collection time of 1 second was used. The eluted peptides were then cleaned up using the stage-tip protocol described above. Similarly, these samples were chromatographically separated and injected onto an Orbitrap Exploris 480 using the same methods described above.

\section{Comparison of full TMT10 plex experiments manual vs. automated}

To evaluate the performance of the automated UbiFast protocol compared to the manual UbiFast protocol using the same PTMScan HS magnetic bead kit we designed three parallel isobaric labeling experiments using TMT10 reagent. For each experiment there were ten process replicates, where each process replicate was $500 \mu \mathrm{g}$ of dried Jurkat peptides. One of these experiments was performed using the UbiFast protocol ${ }^{15}$. The other two experiments were performed on the optimized automated UbiFast protocol described below. These experiments were identical except one labeled with the TMT10 reagent for 10 minutes and the other labeled with TMT10 reagent for 20 minutes to assess the effect of increased labeling time on labeling efficiency.

For all three experiments the desalted sample was transferred to an HPLC vial, frozen, and dried via vacuum centrifugation. Dried peptides were then reconstituted in 9 $\mu \mathrm{l} 3 \% \mathrm{ACN} / 0.1 \% \mathrm{FA}$ and analyzed with back-to-back injections of $4 \mu \mathrm{l}$ using the same liquid chromatography parameters as above. Ion acquisition was performed with an 
Automating UbiFast for Multiplexed Ubiquitin Enrichment

Orbitrap Exploris 480 (Thermo Fisher Scientific) mass spectrometer in line with a FAIMS Pro ${ }^{\mathrm{TM}}$ Interface (Thermo Fisher Scientific). The FAIMS device was operated in standard resolution mode at $100^{\circ} \mathrm{C}$, utilizing the compensation voltages (CVs) of -40,60 , and -80 for the first injection followed by a second injection with CVs of $-40,-50$, and -70. Remaining parameters were identical to the previous manual TMT-labeling comparison experiment, with the exceptions of utilizing a top 10 method for MS2 spectra collection and an MS2 resolution of 45,000.

\section{UbiFast protocol using HSmag anti-K- $\varepsilon-G G$ reagent}

For the manual UbiFast comparison a 10-plex experiment with $500 \mu \mathrm{g}$ Jurkat peptide input per channel was performed. $5 \mu \mathrm{L}$ magnetic beads were washed and aliquoted into $101.5 \mathrm{ml}$ tubes, and K-ع-GG peptide enrichment was performed as described above. After IP, flow-throughs were collected and saved, all samples were washed with $1 \mathrm{ml} 1 \mathrm{X}$ HS IAP Wash Buffer followed by $1 \mathrm{ml} \mathrm{HPLC} \mathrm{H}_{2} \mathrm{O}$, inverting 5 times before centrifuging for $\sim 5 \mathrm{~s}$ at 2,000 $\mathrm{xg}$ and removing the supernatant on a magnetic rack. TMT labeling and quenching was performed identically to the previous single channel experiment, using a full TMT10 ${ }^{\mathrm{TM}}$ 10-plex. Samples were washed first with $1.3 \mathrm{ml}$ followed by $1.5 \mathrm{ml}$ 1X HS IAP Wash Buffer. After washing, each of the 10 TMT-labeled samples were individually resuspended in $90 \mu \mathrm{l} 1 \mathrm{X}$ HS IAP Wash Buffer and combined into a single $1.5 \mathrm{ml}$ tube. This combined sample was mixed by inverting $\sim 5$ times and concentrated by spinning in a benchtop centrifuge at 2,000 x $\mathrm{g}$ for 5-10 s. Beads were allowed to settle on a magnetic rack and the supernatant was slowly aspirated. The 10 original IP tubes were serially washed with $1.5 \mathrm{ml} 1 \mathrm{X}$ HS IAP Wash 
Automating UbiFast for Multiplexed Ubiquitin Enrichment

Buffer to collect any remaining beads and this wash was added to the final sample. Two washes with $1.5 \mathrm{ml}$ HPLC water were performed before eluting TMT-labeled K- $\varepsilon-G G$ peptides with $150 \mu \mathrm{l} 0.15 \%$ TFA for $10 \mathrm{~min}$ at RT, tapping the tube every $2-3 \mathrm{~min}$ to resuspend the beads. The eluate was collected by spinning the sample briefly, setting the tube in a magnetic rack, and slowly pipetting the supernatant onto a StageTip preconditioned as described above. This elution step was repeated once and the sample was StageTip desalted in a manner identical to previous experiments.

\section{Optimized automated UbiFast protocol using HSmag anti-K- $\varepsilon-G G$ reagent}

The automated UbiFast experiments were performed as follows: $5 \mu$ of magnetic bead slurry were aliquoted into 10 wells of a 96-well KingFisher plate. $500 \mu \mathrm{g}$ of dried Jurkat peptides were reconstituted in $250 \mu$ of HS IAP Bind Buffer with $0.01 \%$ CHAPS, placed in a bath sonicator for 2 minutes and clarified by centrifugation for 5 minutes at $10,000 \times \mathrm{g}$. The peptide solution was then pipetted into individual wells in a 96-well KF plate containing HSmag anti-K-ع-GG. The plate was covered with aluminum sealing film (Axygen) and rotated end-over-end for 1 hour at $4^{\circ} \mathrm{C}$.

The plate containing peptides and HSmag anti-K-ع-GG was then moved to the KF. Immediately prior to beginning the method on the KF, $400 \mu \mathrm{g}$ of TMT reagent in 10 $\mu \mathrm{ACN}$ was pipetted into corresponding wells of a 96 -well KF plate. Then $190 \mu$ l of 100 mM HEPES were added to the TMT containing wells. The KF method for TMT labeling consists of 7 steps (Supplemental Figure 2). Step 1 collects the beads from the incubation plate containing the Jurkat peptides and the magnetic beads after mixing at medium speed for 30 seconds. Step 2 washes the beads with $250 \mu$ l of modified HS IAP 
Automating UbiFast for Multiplexed Ubiquitin Enrichment

Wash Buffer [50\% ACN / 50\% HS IAP Wash Buffer with 0.01\% CHAPS] for 1 minute. Step 3 washes the beads with $250 \mu$ of 1 X PBS with $0.01 \%$ CHAPS for 1 minute. Step 4 is on-antibody TMT labeling with $400 \mu \mathrm{g}$ of TMT reagent per channel and either 10 or 20 minutes of labeling time. Step 5 is quenching with $250 \mu$ of $2 \%$ hydroxylamine for 2 minutes. Step 6 is a final wash with $250 \mu$ of the modified HS IAP Buffer for 1 minute. The final step of the KingFisher Flex protocol is to mix the beads in $100 \mu$ of 1 X PBS for 1 minute and then the beads are left in the PBS. For all steps the mixing for the first 15 seconds of the step is set to bottom mix and the remaining time is set to fast. Also, a collect count of 5 and collection time of 1 second was used for all steps. The peptides in PBS were then combined and transferred to a $1.7 \mathrm{~mL}$ eppendorf tube and placed on a magnetic rack for 10 seconds. The PBS was removed and $100 \mu$ of $0.15 \%$ TFA was added to the beads. This elution was repeated once more and both eluates were loaded onto a stage-tip as described previously.

\section{Evaluation of running multiple automated UbiFast experiments simultaneously}

To evaluate increased throughput by automatically and simultaneously enriching multiple TMT plexes, 40 replicates of 500 ug dried peptides were enriched for $\mathrm{K}-\varepsilon-\mathrm{GG}$ and labeled as 4 x TMT10 plexes. All steps were carried out in parallel, using the optimized automated UbiFast workflow described above. Briefly, 5 uL of PBS-washed HSmag anti-K- $\varepsilon-G G$ bead slurry was aliquoted into $4 \times 10$ adjacent wells of a 96 -well KF plate. Dried 500 ug aliquots of A375 melanoma cell line peptides were each reconstituted in 250 uL of HS IAP Bind Buffer with $0.01 \%$ CHAPS, vortexed and shook at $1200 \mathrm{rpm}$ for $5 \mathrm{~min}$, and clarified by centrifugation for 5 minutes at 10,000 $\mathrm{g}$. The peptide solutions were then pipetted into each well containing the magnetic bead slurry. 
Automating UbiFast for Multiplexed Ubiquitin Enrichment

The plate was covered with aluminum film and rotated end-over-end for 1 hour at $4{ }^{\circ} \mathrm{C}$. After incubation the plate was processed on the KF where washing, on-antibody TMT labeling and collection of beads in PBS occured. Each set of K- $\varepsilon-G G$ enriched peptides were combined by TMT10 plex into $1.7 \mathrm{~mL}$ Eppendorf tubes and placed in a magnetic rack for 10 seconds. The PBS was removed and $100 \mathrm{uL} 0.15 \%$ TFA was added to the beads to elute. The elution was repeated once and both eluates were loaded onto stage-tips as previously described. Desalted samples were analyzed via FAIMS-LCMS/MS as previously described.

\section{Processing and analysis of comparative reference tissue}

Processing of WHIM2 and WHIM16 patient-derived xenografts (PDX) for ubiquitylome analysis was described previously ${ }^{15}$. Briefly, frozen tissue from each model was lysed, digested and aliquoted into $500 \mu \mathrm{g}$ aliquots. Five replicates of WHIM2 and 5 replicates of WHIM16 were included in a single TMT10-plex experiment. The digested tissue was processed using the optimized automated UbiFast protocol and LC/MS analysis was performed exactly as detailed in the previous section.

\section{Data Analysis}

Mass spectrometry data was processed using Spectrum Mill (Rev BI.07.04.210, Agilent Technologies). For all samples, extraction of raw files retained spectra within a precursor mass range of 600-6000 Da and a minimum MS1 signal-to-noise ratio of 25. MS1 spectra within a retention time range of $+/-45 \mathrm{~s}$, or within a precursor $\mathrm{m} / \mathrm{z}$ tolerance of $+/-1.4 \mathrm{~m} / \mathrm{z}$ were merged. MS/MS searching was performed against either a 
Automating UbiFast for Multiplexed Ubiquitin Enrichment

human RefSeq database for Jurkat samples or a human and mouse RefSeq database for analysis of PDX samples. Digestion parameters were set to "trypsin allow P" with an allowance of 4 missed cleavages. The MS/MS search included fixed modification of carbamidomethylation on cysteine. For TMT quantitation experiments TMT10 was searched using the partial-mix function. Variable modifications were acetylation of the protein N-terminus, oxidation of methionine and remnant GG on lysine. Restrictions for matching included a minimum matched peak intensity of $40 \%$ and a precursor and product mass tolerance of $+/-20 \mathrm{ppm}$. Peptide matches were validated using a maximum false discovery rate (FDR) threshold of $1.2 \%$. TMT10 reporter ion intensities were corrected for isotopic impurities in the Spectrum Mill protein/peptide summary module using the afRICA correction method which implements determinant calculations according to Cramer's Rule 31 .

\section{Experimental Design and Statistical Rationale}

For the statistical analysis of PDX models, each protein ID was associated with a log2-transformed expression ratio for every sample condition over the median of all sample conditions. After median normalization, a 2-sample moderated T test was performed on the data to compare treatment groups using an internal R-Shiny package based in the limma library. P-values associated with every protein were adjusted using the Benjamini-Hochberg FDR approach ${ }^{32}$. 
Automating UbiFast for Multiplexed Ubiquitin Enrichment

\section{Results}

\section{Comparison of magnetic- and agarose-bead antibody reagents for manual enrichment of K-ع-GG peptides}

The PTMScan® HS Ubiquitin/SUMO Remnant Motif (K-ع-GG) Kit (\#59322)

contains the same monoclonal antibody as the original PTMScan Ubiquitin Remnant (K$\varepsilon$-GG Kit) (\#5562), with the key difference that the new kit contains magnetic beads instead of agarose and bind and wash buffers have been optimized to maximize sensitivity and specificity of K-ع-GG peptide enrichment. The antibody is coupled to the beads using chemistry that does not affect the epitope binding regions. Since this HSmag anti-K-ع-GG formulation does not require an initial chemical cross-linking step to covalently couple the antibody to affinity beads, 1-2 days of procedural time are saved $^{9,23,24}$. The magnetic bead formulation also provides the means to transfer ubiquitin enrichment protocols to a magnetic particle processor for automating and increasing the throughput of sample handling steps.

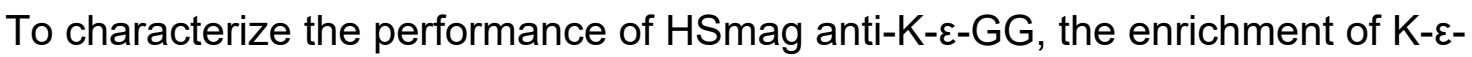
GG peptides was directly compared to enrichment with agarose-bead anti-K-ع-GG antibody in a label-free manner by LC-MS/MS. Enrichment of K- $\varepsilon-G G$ peptides was completed in triplicate from $500 \mu \mathrm{g}$ of tryptic peptides derived from Jurkat cells using 5

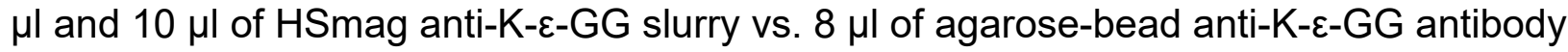
and enriched peptides were analyzed by LC-MS/MS (Supplemental Figure 1 and

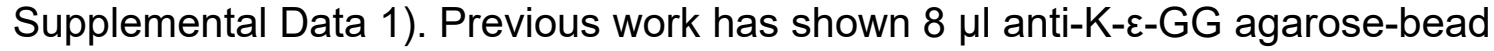

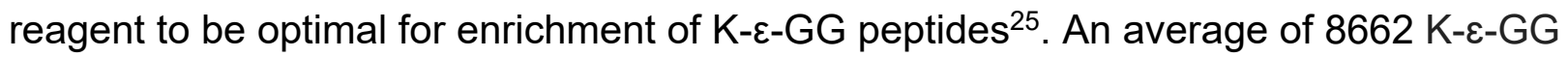


Automating UbiFast for Multiplexed Ubiquitin Enrichment

PSMs were identified with $5 \mu \mathrm{l}$ of HSmag anti-K- $\varepsilon-G G, 9284 \mathrm{~K}-\varepsilon-G G$ PSMs were

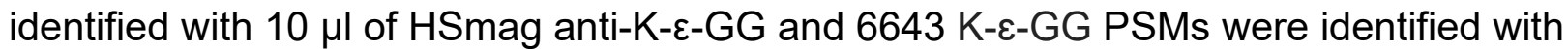

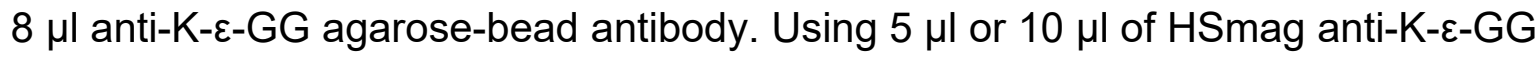
improved recovery of K- $\varepsilon-G G$ PSMs compared to $8 \mu \mathrm{l}$ anti-K- $\varepsilon-G G$ agarose-bead antibody by $30 \%$ and $39 \%$, respectively. The relative yield, or the percentage of K- $\varepsilon-G G$ PSMs identified relative to the total number of PSMs identified in the sample, was $51 \%$

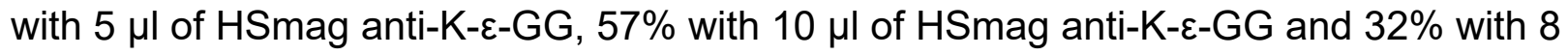

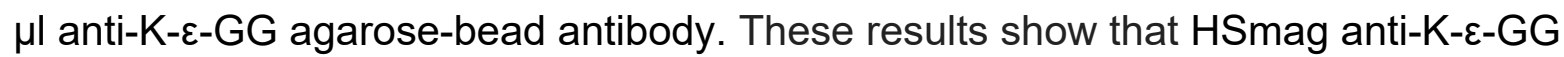

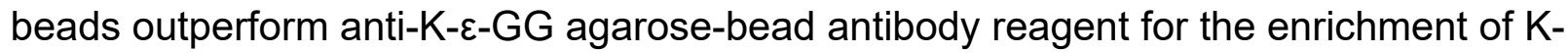
$\varepsilon$-GG peptides using as little as $5 \mu$ of magnetic beads. Although $10 \mathrm{ul}$ of $\mathrm{HS}$ mag antiK- $\varepsilon-G G$ beads minimized variability, it provided only a relatively small increase in the number of identified K- $\varepsilon$-GG PSMs vs. the use of $5 \mu \mathrm{l}$ of HSmag anti-K- $\varepsilon-G G$ slurry, indicating that the lower amount of beads is efficient and cost effective.

\section{Evaluation of on-antibody TMT labeling using HSmag anti-K- $\varepsilon-G G$}

The UbiFast method utilizes on-antibody TMT labeling for isobaric labeling experiments ${ }^{15}$. To evaluate the performance of on-antibody TMT labeling using HSmag anti-K- $\varepsilon-G G$ antibody, Jurkat peptides (500 $\mu$ g in triplicate) were enriched using HSmag

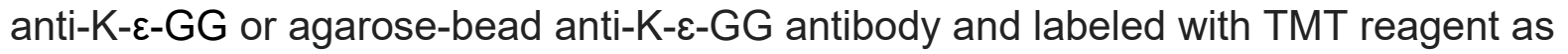
previously described ${ }^{15}$ (Supplemental Data 2). For this evaluation, a single channel of TMT reagent was used to label each replicate. We identified an average of 8008 and $5289 \mathrm{~K}-\varepsilon-\mathrm{GG}$ PSMs with $5 \mu \mathrm{L}$ magnetic and $8 \mu \mathrm{L}$ agarose-bead antibody reagent, respectively (Supplemental Fig 1b). 
Automating UbiFast for Multiplexed Ubiquitin Enrichment

To analyze the efficiency of TMT labeling we evaluated the number of K- $\varepsilon-G G$ PSMs fully TMT labeled, partially TMT labeled and unlabeled. A peptide is considered fully labeled if the $\mathrm{N}$-terminal amine group $\left(-\mathrm{NH}_{2}\right)$ and the $\varepsilon$-amino group on lysines (if present) have reacted with the TMT reagent. Partially labeled PSMs have one amine group that has reacted with TMT and at least one amine group that has not reacted with

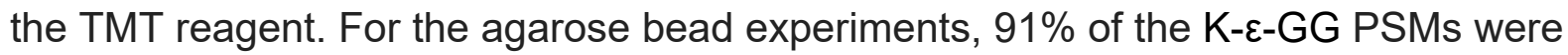
fully labeled and $5 \%$ were partially labeled. For the magnetic bead experiments, $91 \%$ of the K-ع-GG PSMs were fully labeled and 5\% were partially labeled (Supplemental Fig

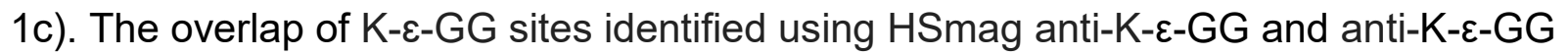
antibody was $>52 \%$ (Supplemental Figure 1d). Each method also identified a subset of distinct Ub sites. These results demonstrate that on-antibody TMT labeling works

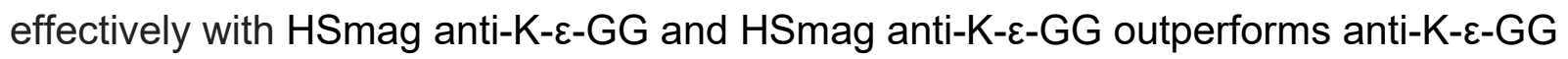
antibody for identifying K-ع-GG peptides following enrichment and on-antibody TMT labeling.

\section{Optimization of automated workflow for the UbiFast method}

To increase the throughput and reproducibility of the UbiFast method, we sought to reduce the number of manual sample processing steps in the method by automating the HSmag anti-K- $\varepsilon-G G$ washing and on-antibody TMT labeling steps using the KingFisher Flex (KF), a magnetic bead processor capable of processing 96-well plates. The KF operates by using magnetic rods to collect magnetic beads from a 96-well plate and transfer them into another 96 -well plate. Following offline K- $\varepsilon-G G$ peptide capture in a 96 well plate, the plate was loaded onto the KF where antibody beads were washed 
Automating UbiFast for Multiplexed Ubiquitin Enrichment

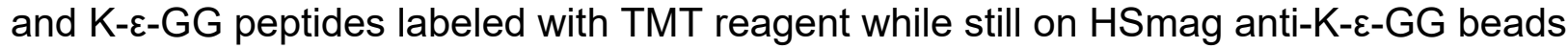
(Supplemental Figure 2).

First, we evaluated how efficiently HSmag anti-K-ع-GG transfer from one 96-well plate to a second 96-well plate on the KF. Previous work showed that adding low concentrations of CHAPS to buffers prevents magnetic beads from sticking to the plates and being lost during KF processing steps ${ }^{26}$. We compared automated UbiFast with and without addition of $0.01 \%$ CHAPS to wash buffers and found that CHAPS significantly improves the automated protocol, identifying 1.9-fold more K- $\varepsilon-G G$ peptides compared to no CHAPS addition (3329 vs 1752 K- $\varepsilon-G G$ PSMs) (Supplemental Figure 3a, Supplemental Data 3). Omitting CHAPS from wash buffers clearly resulted in residual HSmag anti-K- $\varepsilon-G G$ beads being left in the plates after the completion of the KF run. These experiments confirmed that CHAPS improves the movement of magnetic beads in this protocol, increasing the number of K-ع-GG PSMs by $89 \%$ and decreasing variability due to loss of beads and should be added to all buffers used on the KF platform.

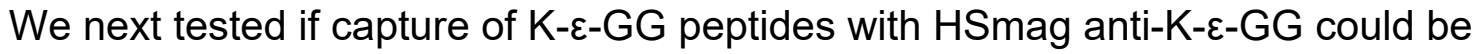
completed on the KF. In the manual protocol, the enrichment step is complete at $4^{\circ} \mathrm{C}$ with end-over-end rotation ${ }^{9}$. The KF does not have a cooling unit and cannot rotate plates end-over-end. Therefore we sought to confirm whether the enrichment step should be performed offline as is done in the manual protocol or on the KF at RT with mixing. To evaluate the capture step, two 96-well KF plates were prepared, each with three wells containing $500 \mu \mathrm{g}$ Jurkat peptides and the HSmag anti-K- $\varepsilon-G G$. One 96-well plate was rotated end-over-end in a cold room at $4^{\circ} \mathrm{C}$ and the other plate was incubated 
Automating UbiFast for Multiplexed Ubiquitin Enrichment

on the KF at room temperature with mixing set to 'medium' (Supplemental Figure 3b, Supplemental Data 4). A large drop in PSMs was observed when the incubation was performed at RT on the KingFisher, identifying 3308 K- $\varepsilon-G G$ PSMs compared to 6215 K- $\varepsilon-G G$ PSMs with end-over-end rotation at $4^{\circ} \mathrm{C}$. These results indicate that offline endover-end rotation at $4^{\circ} \mathrm{C}$ is preferable for binding of $\mathrm{K}-\varepsilon-\mathrm{GG}$ peptides to the $\mathrm{HSmag}$ antiK-ع-GG. Performing this step offline will not add any additional time or labor since the samples and beads are already in the 96-well plate, however, future studies could explore placing the KingFisher in a cold room at $4^{\circ} \mathrm{C}$.

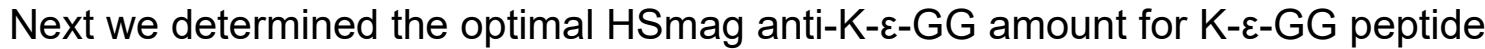
enrichment on the KF. Previous work showed that decreasing the amount of agarosebead anti-K- $\varepsilon-G G$ antibody increased the relative yield and overall recovery of $K-\varepsilon-G G$ peptides $^{9}$. Given the previous findings, we titrated the amount of HSmag anti-K- $\varepsilon-G G$ to determine which bead volume would produce the highest overall number and the highest relative yield of $\mathrm{K}-\varepsilon-\mathrm{GG}$ peptides. We performed quintuplicate enrichments using $5 \mu \mathrm{L}, 10 \mu \mathrm{L}$ and $20 \mu \mathrm{L}$ of HSmag anti-K- $-\varepsilon-G \mathrm{G}$ and compared the overall number of K-ع-GG PSMs and relative yield (Supplemental Figure 3c, Supplemental Data 5). The number of identified K- $\varepsilon-G G$ PSMs was 6519,5261 and 4540 for 5,10 and $20 \mu \mathrm{L}$ of

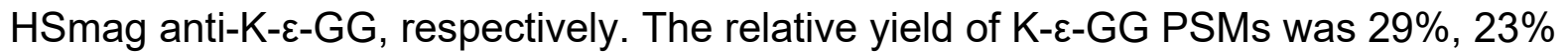
and $20 \%$ for 5,10 and $20 \mu \mathrm{L}$ of HSmag anti-K- $-\mathrm{G}-\mathrm{GG}$, respectively. Enrichment with $5 \mu \mathrm{L}$

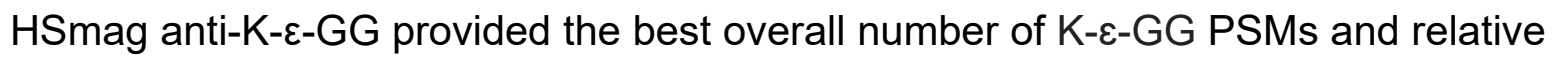
yield of K-ع-GG PSMs.

We noted that the relative yield of K-ع-GG PSMs to total PSMs was much lower for $\mathrm{HSmag}$ anti-K- $\varepsilon-G G$ processing on the KF relative to manual processing (29\% vs 
Automating UbiFast for Multiplexed Ubiquitin Enrichment

$51 \%)$. We hypothesized that the decrease in relative yield of $\mathrm{K}-\varepsilon-\mathrm{GG}$ peptides could be due to insufficient bead washing because the volume of each wash step was reduced from $1 \mathrm{~mL}$ in the manual protocol to $250 \mu \mathrm{L}$ on the $\mathrm{KF}$ to accommodate the maximum volume of the 96 -well plate. To reduce the number of non-K- $\varepsilon-G G$ peptides resulting from automated processing, $50 \% \mathrm{ACN}$ was added to the wash buffer to increase stringency. To evaluate the utility of ACN addition to the wash buffer, we performed a label-free experiment where K-ع-GG peptides were enriched from $500 \mu \mathrm{g}$ Jurkat peptides where bead washing was completed with or without $50 \%$ ACN added to the HS IAP Wash Buffer (Supplemental Figure 3d, Supplemental Data 6). Addition of 50\% ACN to the washing buffer increased both the relative yield of $\mathrm{K}-\varepsilon-\mathrm{GG}$ peptides and the absolute number of identified K- $\varepsilon-G G$ peptides. An average of $7210 \mathrm{~K}-\varepsilon-G G$ PSMs with $57 \%$ relative yield and 5694 K- $\varepsilon-G G$ PSMs with $29 \%$ relative yield were identified with and without $50 \%$ ACN added to the wash buffer, respectively. Taken together, we find that use of $5 \mu \mathrm{L}$ of HSmag anti-K- - -GG bead slurry and inclusion of $0.01 \%$ CHAPS and $50 \% \mathrm{ACN}$ in wash buffers provides the best performance of the UbiFast method on the $\mathrm{KF}$.

\section{Manual vs. automated TMT 10-plex experiment}

The Ubifast method is designed to be used with multiplexed samples ${ }^{15}$. To evaluate the performance of the automated UbiFast workflow, we carried out a head-tohead comparison using HSmag anti-K- $\varepsilon-G G$ where both automated and manual UbiFast methods were used to analyze 10 process replicates of peptides from Jurkat cells, with 500 ug peptide input per replicate using a different TMT10 reagent for each replicate. 
Automating UbiFast for Multiplexed Ubiquitin Enrichment

Due to the additional time it takes to prepare and aliquot TMT reagents in a 96-well plate for multiplexed experiments, we performed two independent automated TMT10 UbiFast experiments comparing 10 minutes and 20 minutes on-antibody TMT labeling time to determine if a longer TMT labeling time is needed due to reagent hydrolysis during preparation steps (Figure 2a). For manual and automated experiments, we

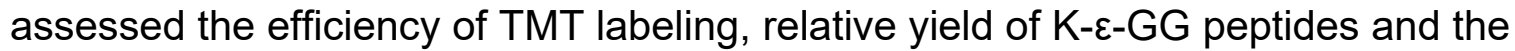
variability between differentially labeled TMT channels (Figure $2 b, 2 c, 2 d$, Supplemental Data 7).

Using the manual UbiFast protocol, we identified 16,141 distinct K- $\varepsilon-G G$ peptides with $97 \%$ of peptides being labeled with TMT and a relative yield of $75 \%$. The automated UbiFast experiment identified 21,468 with $88 \%$ relative yield and 21,601 distinct K- $\varepsilon-G G$ peptides with $87 \%$ relative yield using 10 and 20 minutes of on-antibody TMT labeling time, respectively. Increasing on-antibody TMT labeling time from 10 min to 20 min increased the TMT labeling efficiency from $95 \%$ to $97 \%$. The reproducibility of automated UbiFast experiments was higher than manual UbiFast, with median coefficients of variation across process replicates of $20 \%, 19 \%, 25 \%$ for automated UbiFast 10min, automated UbiFast 20 min, manual UbiFast, respectively. These data

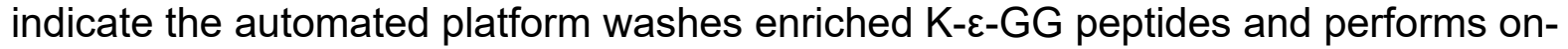
antibody TMT labeling with high reproducibility, eliminating the need for manual processing. 
Automating UbiFast for Multiplexed Ubiquitin Enrichment

Automating the UbiFast method reduces the hands-on time for processing a single multiplexed TMT experiment, but the greatest impact would be in the ability to scale the number of multiplexed TMT experiments that could be processed simultaneously. To evaluate the effects of processing multiple TMT experiments, we designed an experiment in which 4 x TMT10 experiments were processed in parallel (Figure 3a). Briefly, a batch of peptides digested with trypsin from A375 cells were aliquoted into $40 \times 500 \mu \mathrm{g}$ aliquots. The 40 aliquots were split into 4 groups of 10 and processed on the KF as described above with each group of 10 aliquots processed as separate TMT10 experiments. To assess performance across all 4 experiments, we

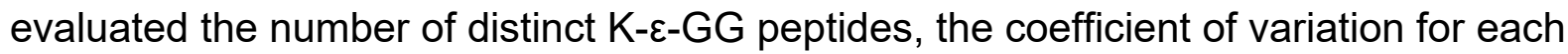
experiment and the labeling efficiency (Figure 3b, 3c, 3d, Supplemental Data 8). We identified 17,718, 16,704, 17,668 and 16,862 distinct K-ع-GG peptides in experiments 1 through 4 , respectively. The median coefficient of variation for each plex was $20.8,19.8$, 21.2 and 20.8 and the percentage of fully labeled K- - -GG PSMs was $95 \%-96 \%$ for all 4 plexes. These results indicate that the automated UbiFast protocol enables multiple multiplexed TMT experiments to be processed simultaneously with high reproducibility, saving up to 2 hours of laboratory time for each additional TMT experiment.

\section{Application of automated UbiFast with HSmag anti-K- $\varepsilon-G G$ to analysis of tumor}

\section{samples}

Tumor tissues of two breast cancer patient-derived xenograft (PDX) models, basal subtype (WHIM2) and luminal subtype (WHIM16) have been extensively characterized in the proteogenomic space, having been shown to harbor subtype- 
Automating UbiFast for Multiplexed Ubiquitin Enrichment

specific signatures ${ }^{15,27,28}$. To evaluate our automated UbiFast method for profiling ubiquitylomes of small amounts of tumor tissue, we applied the workflow to enrich ubiquitylated peptides from five replicates each of WHIM2 and WHIM16 using $0.5 \mathrm{mg}$ of peptide per sample in a TMT10-plex experiment (Figure 4a). The correlation of replicates within the automated UbiFast dataset employing HSmag anti-K-ع-GG beads was high, with median Pearson correlations of 0.76 and 0.73 for basal and luminal

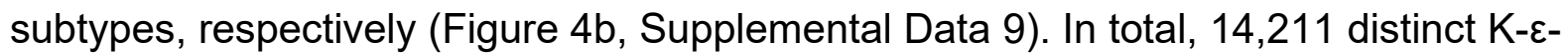
GG peptides were identified and quantified using this method. Ubiquitylation data was compared to previously published results acquired on the sample PDX models, but employing UbiFast in a manual mode using agarose K- $\varepsilon-G G$ antibody to evaluate the overlap of K- $\varepsilon-G G$ sites and conservation of canonical biological differences between basal and luminal breast cancer models. Among these identified K-E-GG sites, we see a high degree of overlap at the site level (61\%) between datasets (Figure 4c). To assess whether biological changes were conserved across UbiFast workflows, we performed gene set enrichment analysis (GSEA) on both datasets using the GO Biological Process Molecular Signature Database ${ }^{29,30}$. Gene Sets which were significantly changing in both datasets are shown in Figure 4d with the top 5 gene sets enriched in the luminal and basal subtypes from the Udeshi et al. 2020 study highlighted. It is clear that the gene sets which were significantly enriched with the automated method show the same trends as those enriched by Udeshi et al. 2020. 
Automating UbiFast for Multiplexed Ubiquitin Enrichment

\section{Discussion}

Here we present an automated UbiFast workflow using magnetic-bead K-ع-GG antibody and a robotic magnetic particle processor with a 96 well plate format to increase sample analysis throughput of the UbiFast method while decreasing labor required and chances for human error (Figure 1). Removing the need for a highly trained person to perform these enrichments should make ubiquitylation site profiling accessible to more laboratories. Using the manual implementation of the UbiFast method, it takes more than 1 day of processing time to prepare 10 samples $(1 \times 10$-plex experiment) for injection on the mass spectrometer because the antibody must first be cross-linked to agarose beads followed by quality control ${ }^{28}$. Magnetic HSmag anti-K- $\varepsilon$ GG supplied in the PTMScan® HS Ubiquitin/SUMO Remnant Motif (K-ع-GG) kit are provided with the antibody already linked to the magnetic beads and do not require additional cross-linking, simplifying the method and reducing the total processing time to 3 hours per 1 x 10-plex experiment. Importantly, the magnetic HSmag anti-K- $\varepsilon-G G$ reagent identified more Ub-peptides with greater reproducibility than the agarose anti-K$\varepsilon-G G$ reagent in TMT experiments. Automation of the UbiFast method on the KF reduces sample processing time per plex from 3 hours to 2 hours and allows for parallel processing of up to $9 \times$ TMT10-plex experiments. Future efforts will be aimed at extending to higher-plex isobaric labeling methods such as TMTPro (Thermo Fisher), enabling $6 \times 16$-plex experiments to be processed in parallel. The KF platform may be used to automate the enrichment of other PTMs, such as phosphorylated tyrosine and acetylated lysines as magnetic bead reagents become available in the future. 
Automating UbiFast for Multiplexed Ubiquitin Enrichment

\section{Acknowledgements}

This work was supported by the National Cancer Institute (NCI) grants U24CA210986, U01CA214125, and U24CA210979 to S.A.C., Swiss National Science Foundation (SNF) Sinergia grant CRSII5_186405 to S.A.C., Dr. Miriam and Sheldon G. Adelson Medical Research Foundation to S.A.C. and N.D.U. and by a SPARC Award to N.D.U. from the Broad Institute of MIT \& Harvard (\#800373)

\section{Authors contribution}

K.D.R., M.E.O., S.A.C. and N.D.U. conceived the study; K.D.R., M.E.O., and E.J.B. performed experiments. K.D.R., M.E.O., E.J.B., A.J.N., K.A.L., S.S., S.A.C. and N.D.U. contributed to experimental design, data analysis, and data interpretation. K.D.R., M.E.O., S.A.C. and N.D.U. generated figures and wrote the manuscript with input from all authors.

\section{Data availability}

Raw mass spectrometry data will be made publicly available in MassIVE upon acceptance of the manuscript. This article contains supplemental data. 
Automating UbiFast for Multiplexed Ubiquitin Enrichment

\section{References}

1. Hershko, A. \& Ciechanover, A. The ubiquitin system. Annu. Rev. Biochem. 67, 425-479 (1998).

2. Celebi, G., Kesim, H., Ozer, E. \& Kutlu, O. The Effect of Dysfunctional Ubiquitin Enzymes in the Pathogenesis of Most Common Diseases. Int. J. Mol. Sci. 21, (2020).

3. Kortuem, K. M. \& Stewart, A. K. Carfilzomib. Blood 121, 893-897 (2013).

4. Hideshima, T. et al. The proteasome inhibitor PS-341 inhibits growth, induces apoptosis, and overcomes drug resistance in human multiple myeloma cells. Cancer Res. 61, 30713076 (2001).

5. Schapira, M., Calabrese, M. F., Bullock, A. N. \& Crews, C. M. Targeted protein degradation: expanding the toolbox. Nat. Rev. Drug Discov. 18, 949-963 (2019).

6. Akimov, V. et al. UbiSite approach for comprehensive mapping of lysine and N-terminal ubiquitination sites. Nat. Struct. Mol. Biol. 25, 631-640 (2018).

7. Danielsen, J. M. R. et al. Mass spectrometric analysis of lysine ubiquitylation reveals promiscuity at site level. Mol. Cell. Proteomics 10, M110.003590 (2011).

8. Kim, W. et al. Systematic and Quantitative Assessment of the Ubiquitin-Modified Proteome. Mol. Cell 44, 325-340 (2011).

9. Udeshi, N. D., Mertins, P., Svinkina, T. \& Carr, S. A. Large-scale identification of ubiquitination sites by mass spectrometry. Nat. Protoc. 8, 1950-1960 (2013).

10. Na, C. H. et al. Synaptic Protein Ubiquitination in Rat Brain Revealed by Antibody-based Ubiquitome Analysis. J. Proteome Res. 11, 4722-4732 (2012).

11. Xu, G., Paige, J. S. \& Jaffrey, S. R. Global analysis of lysine ubiquitination by ubiquitin remnant immunoaffinity profiling. Nat. Biotechnol. 28, 868-873 (2010).

12. Thompson, A. et al. Tandem Mass Tags: A Novel Quantification Strategy for Comparative 
Automating UbiFast for Multiplexed Ubiquitin Enrichment

Analysis of Complex Protein Mixtures by MS/MS. Anal. Chem. 75, 1895-1904 (2003).

13. Thompson, A. et al. TMTpro: Design, Synthesis, and Initial Evaluation of a Proline-Based Isobaric 16-Plex Tandem Mass Tag Reagent Set. Anal. Chem. 91, 15941-15950 (2019).

14. Li, J. et al. TMTpro reagents: a set of isobaric labeling mass tags enables simultaneous proteome-wide measurements across 16 samples. Nat. Methods 17, 399-404 (2020).

15. Udeshi, N. D. et al. Rapid and deep-scale ubiquitylation profiling for biology and translational research. Nat. Commun. 11, (2020).

16. Emanuele, M. J. et al. Global Identification of Modular Cullin-RING Ligase Substrates. Cell $147,459-474(2011)$.

17. Gendron, J. M. et al. Using the Ubiquitin-modified Proteome to Monitor Distinct and Spatially Restricted Protein Homeostasis Dysfunction. Mol. Cell. Proteomics 15, 2576-2593 (2016).

18. Kronke, J. et al. Lenalidomide Causes Selective Degradation of IKZF1 and IKZF3 in Multiple Myeloma Cells. Science 343, 301-305 (2014).

19. Povlsen, L. K. et al. Systems-wide analysis of ubiquitylation dynamics reveals a key role for PAF15 ubiquitylation in DNA-damage bypass. Nat. Cell Biol. 14, 1089-1098 (2012).

20. Sarraf, S. A. et al. Landscape of the PARKIN-dependent ubiquitylome in response to mitochondrial depolarization. Nature 496, 372-376 (2013).

21. Satpathy, S. et al. Systems-wide analysis of BCR signalosomes and downstream phosphorylation and ubiquitylation. Mol. Syst. Biol. 11, 810 (2015).

22. Wagner, S. A., Satpathy, S., Beli, P. \& Choudhary, C. SPATA2 links CYLD to the TNF- $\alpha$ receptor signaling complex and modulates the receptor signaling outcomes. EMBO J. $\mathbf{3 5}$, 1868-1884 (2016).

23. Rose, C. M. et al. Highly Multiplexed Quantitative Mass Spectrometry Analysis of Ubiquitylomes. Cell Systems 3, 395-403.e4 (2016).

24. Hansen, F. M. et al. Data-independent acquisition method for ubiquitinome analysis reveals 
Automating UbiFast for Multiplexed Ubiquitin Enrichment

regulation of circadian biology. Nat. Commun. 12, 1-13 (2021).

25. Udeshi, N. D. et al. Refined preparation and use of anti-diglycine remnant (K-ع-GG) antibody enables routine quantification of 10,000 s of ubiquitination sites in single proteomics experiments. Mol. Cell. Proteomics 12, 825-831 (2013).

26. Kuhn, E. et al. Interlaboratory evaluation of automated, multiplexed peptide immunoaffinity enrichment coupled to multiple reaction monitoring mass spectrometry for quantifying proteins in plasma. Mol. Cell. Proteomics 11, M111 013854 (2012).

27. Li, S. et al. Endocrine-Therapy-Resistant ESR1 Variants Revealed by Genomic Characterization of Breast-Cancer-Derived Xenografts. Cell Rep. 4, 1116-1130 (2013).

28. Mertins, P. et al. Reproducible workflow for multiplexed deep-scale proteome and phosphoproteome analysis of tumor tissues by liquid chromatography-mass spectrometry. Nat. Protoc. 13, 1632-1661 (2018).

29. Subramanian, A. et al. Gene set enrichment analysis: A knowledge-based approach for interpreting genome-wide expression profiles. Proceedings of the National Academy of Sciences 102, 15545-15550 (2005).

30. Mootha, V. K. et al. PGC-1a-responsive genes involved in oxidative phosphorylation are coordinately downregulated in human diabetes. Nat. Genet. 34, 267-273 (2003).

31. Shadforth, I. P., Dunkley, T. P. J., Lilley, K. S. \& Bessant, C. i-Tracker: For quantitative proteomics using iTRAQTM. BMC Genomics 6, 1-6 (2005).

32. Benjamini, Y. \& Hochberg, Y. Controlling the false discovery rate: A practical and powerful approach to multiple testing. J. R. Stat. Soc. 57, 289-300 (1995). 
Automating UbiFast for Multiplexed Ubiquitin Enrichment

\section{Figures}

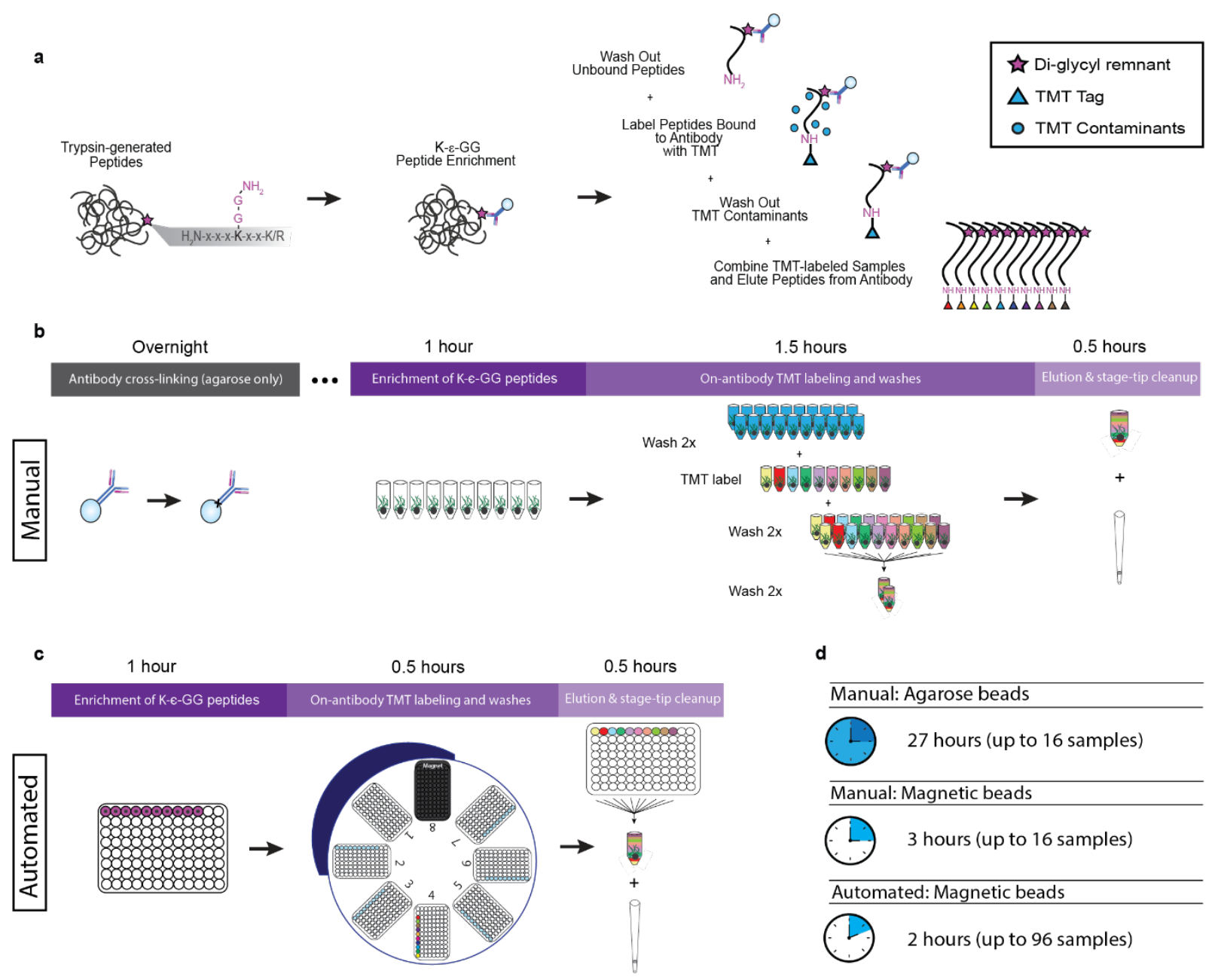

Figure 1 Comparison of methods for on-antibody labeling of K- $\varepsilon-G G$ peptides with

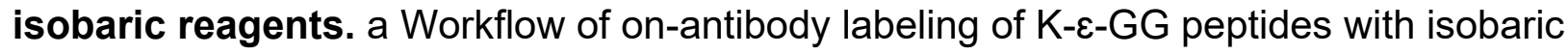
reagents using the UbiFast method. b Manual UbiFast workflow for processing a TMT10 experiment. c Automated workflow of processing samples for a TMT10 experiment using 96-well plates and a KingFisher Flex instrument. 
bioRxiv preprint doi: https://doi.org/10.1101/2021.04.28.441860; this version posted June 23, 2021. The copyright holder for this preprint (which was not certified by peer review) is the author/funder, who has granted bioRxiv a license to display the preprint in perpetuity. It is made available under aCC-BY-NC-ND 4.0 International license.

Automating UbiFast for Multiplexed Ubiquitin Enrichment

a

\begin{tabular}{|c|}
\hline Manual 10-plex \\
\hline K- $\varepsilon$-GG peptide enrichment \\
\hline Wash with HS IAP Wash Buffer+ PBS \\
\hline On-antibody TMT labeling \\
\hline Wash with IAP \\
\hline Combine TMT10-labeled samples \\
\hline Wash with IAP + PBS \\
\hline Elute $\mathrm{K}-\varepsilon-\mathrm{GG}$ peptides \\
\hline
\end{tabular}

Jurkat Cells

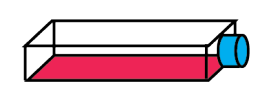

$\downarrow$

Trypsin-digested peptides

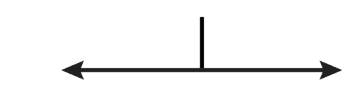

$10 \times 500$ ug $\quad 10 \times 500$ ug

$10 \times 500 \mathrm{ug}$
Automated 10-plex

K-E-GG peptide enrichment

Wash with $50 \% \mathrm{HS} \mathrm{IAP} / 50 \% \mathrm{ACN}+\mathrm{PBS}$ (both with $0.01 \%$ CHAPS)

On-antibody TMT labeling (either 10 or 20 minutes)

Wash with PBS

Combine TMT10-labeled samples (manually)

Elute $\mathrm{K}-\varepsilon$-GG peptides b

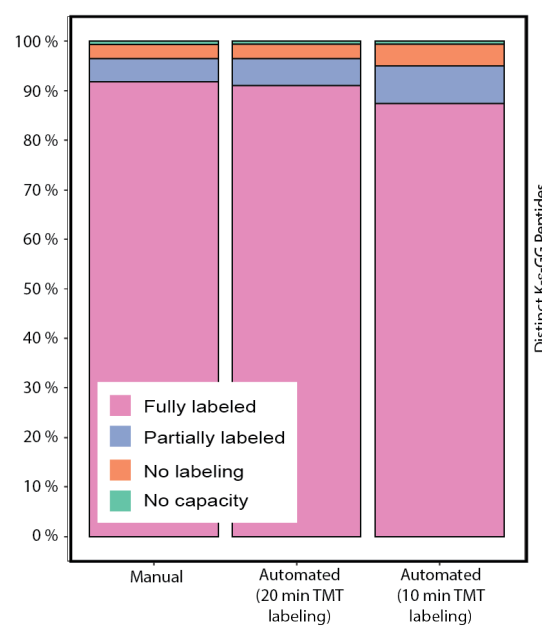

C

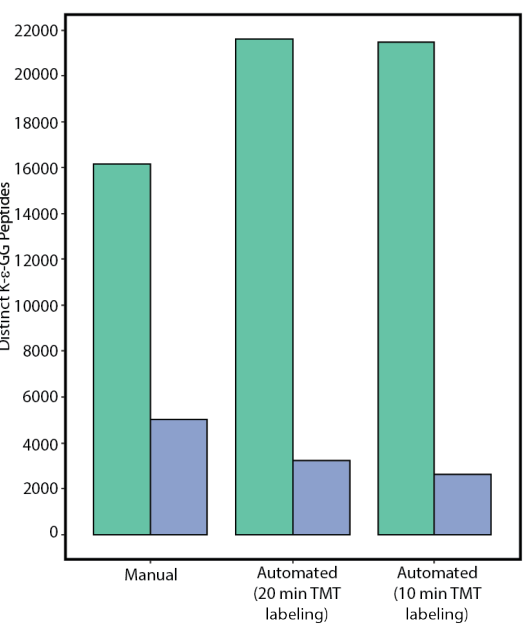

d

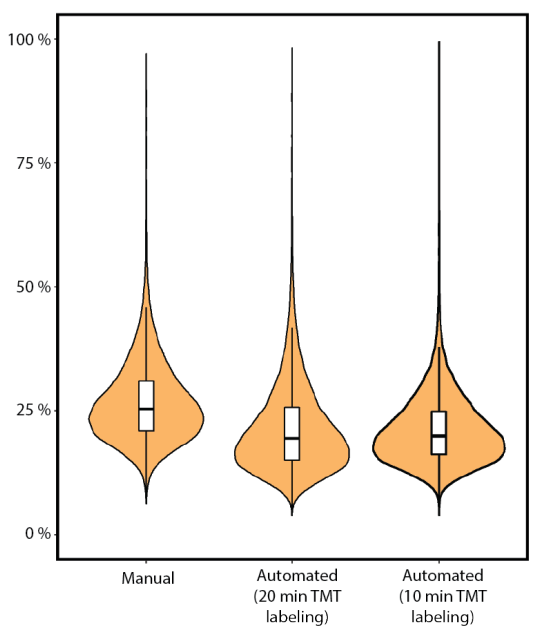

Figure 2 Analysis of K- $\varepsilon-G G$ peptide enrichment in a 10-plex format using

automation vs. manual workflows . a Experimental design of parallel 10-plex ubiquitin enrichment experiments using TMT10 reagents for on-antibody TMT labeling. b

Stacked bar plots show the percentage of fully, partially and unlabeled K-ع-GG PSM c

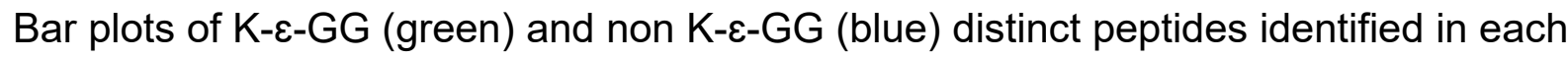
experiment. $\mathbf{d}$ Violin plots show the distribution of CV's between differentially labeled TMT channels for each experiment with the inner box and whisker plots showing the median and interquartile range. 
bioRxiv preprint doi: https://doi.org/10.1101/2021.04.28.441860; this version posted June 23, 2021. The copyright holder for this preprint (which was not certified by peer review) is the author/funder, who has granted bioRxiv a license to display the preprint in perpetuity. It is made available under aCC-BY-NC-ND 4.0 International license.

Automating UbiFast for Multiplexed Ubiquitin Enrichment

a
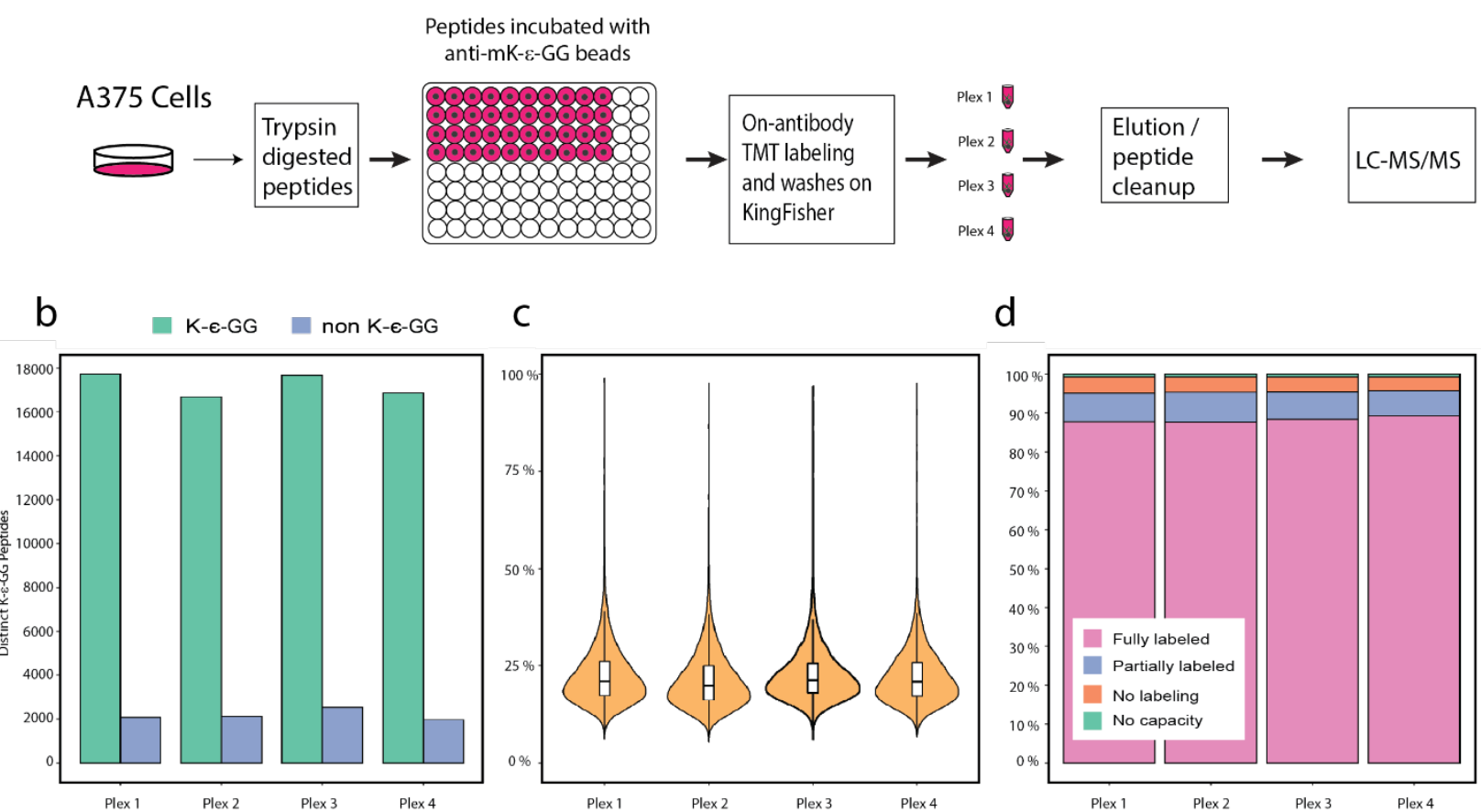

C

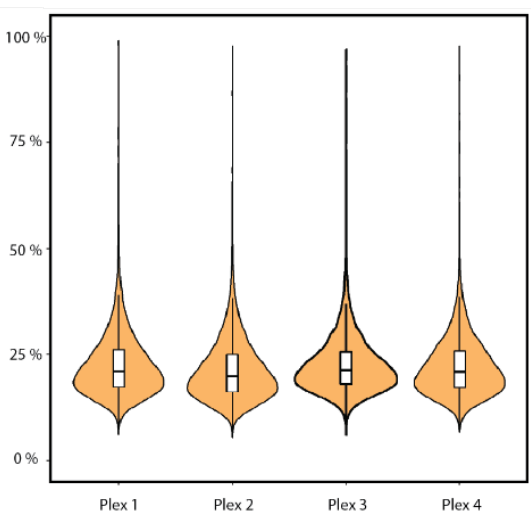

d

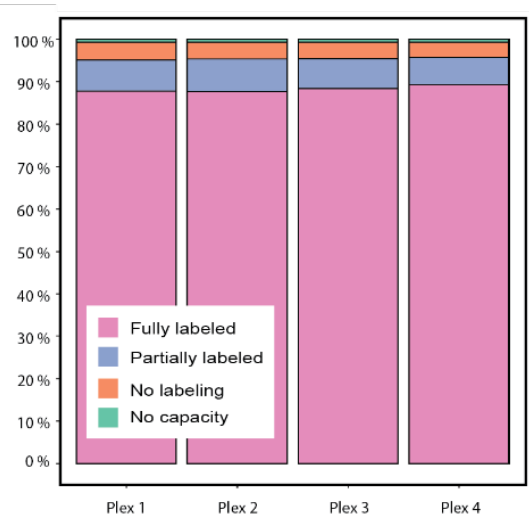

Figure 3 Parallel processing of 4 x TMT10 experiments using the automated

UbiFast method. a Schematic of experimental workflow for processing multiple experiments in parallel. b Stacked bar plots show the percentage of fully, partially and

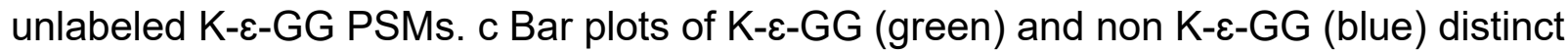
peptides identified in each experiment. $d$ Violin plots show the distribution of CV's between differentially labeled TMT channels for each experiment with the inner box and whisker plots showing the median and interquartile range 
Automating UbiFast for Multiplexed Ubiquitin Enrichment

a

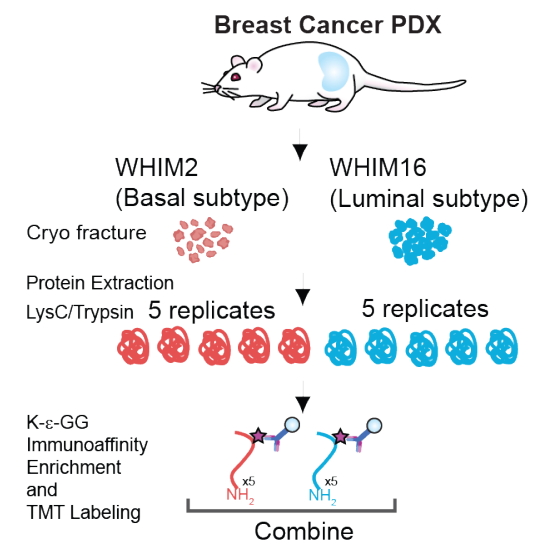

C

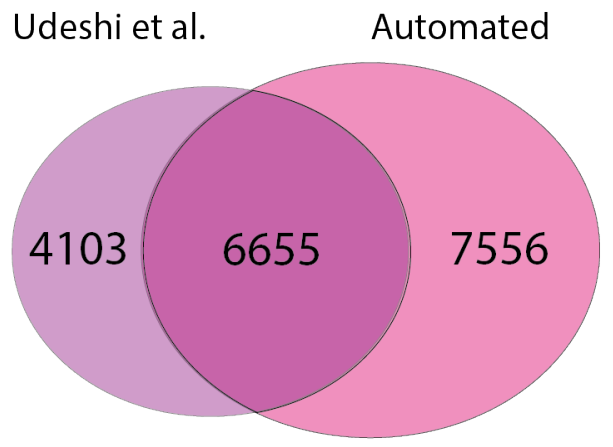

b

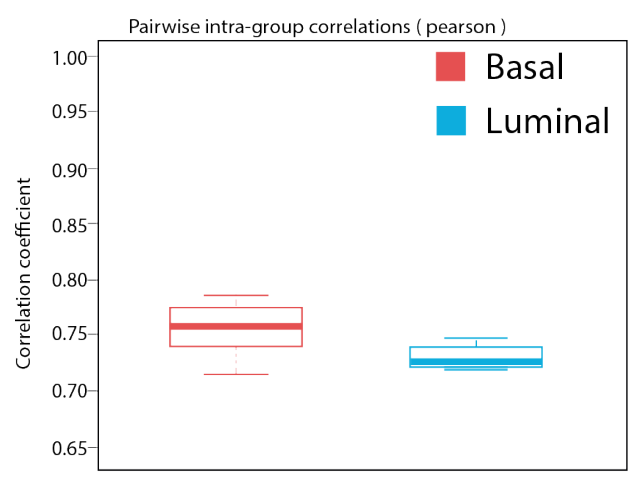

d

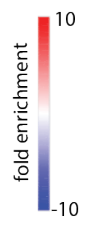

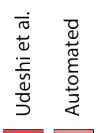

Oxidation and reduction process

Organonitrogen compound biosynthesis process

Small molecule catabolic process

Glucose catabolic process

Monosacharide catabolic process

Morphogenesis of a polarized epithelium

Tissue morphogenesis

Canonical WNT signaling pathway

Regulation of anatomical structure morphogenesis

Regulation of animal organ morphogenesis

Figure 4 Comparison of the ubiquitylomes identified from our automated

workflow vs manually workflow ${ }^{15}$. a Schematic diagram showing experimental design and process used to enrich K-E-GG modified peptides from the Luminal and Basal breast cancer PDX models. b Venn Diagram showing overlap of K- $\varepsilon$-GG sites identified in each dataset. c Box of whisker plots of Pearson correlation between replicates of Basal and Luminal PDX models for each dataset. d Heatmap of all genesets present in both the Udeshi et al dataset (left) and the current, automated dataset (right) with the top 5 most enriched gene sets in the Udeshi et al dataset highlighted. 
bioRxiv preprint doi: https://doi.org/10.1101/2021.04.28.441860; this version posted June 23, 2021. The copyright holder for this preprint (which was not certified by peer review) is the author/funder, who has granted bioRxiv a license to display the preprint in perpetuity. It is made available under aCC-BY-NC-ND 4.0 International license.

\section{Automating UbiFast for Multiplexed Ubiquitin Enrichment}

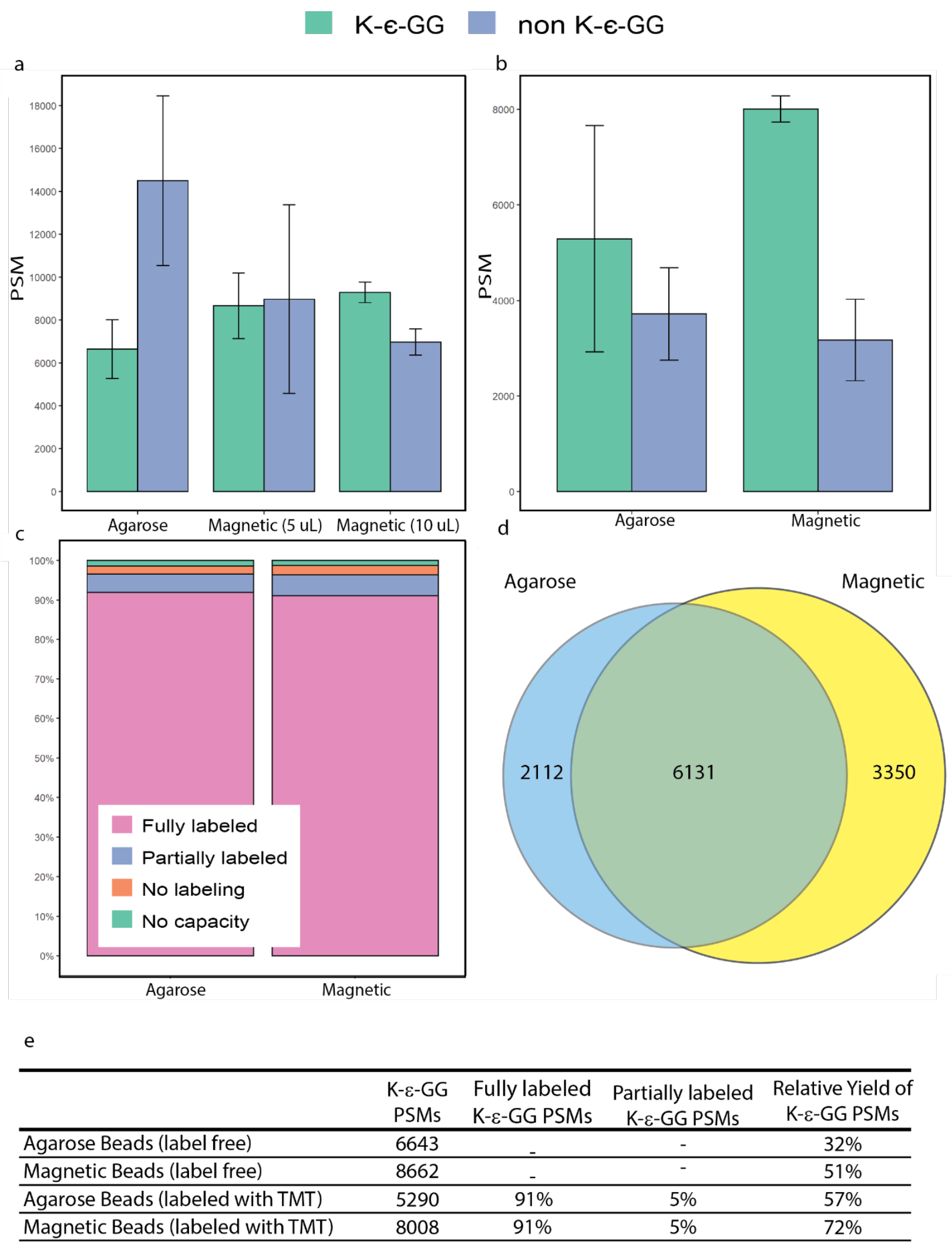


Automating UbiFast for Multiplexed Ubiquitin Enrichment

Supplemental Figure 1. Comparison of K- $\varepsilon-G G$ peptide enrichments with antibody coupled to either agarose or magnetic beads. a Bar plots show identification of K- $\varepsilon-G G$ and non K-ع-GG PSMs for label free enrichments with agarose and magnetic beads

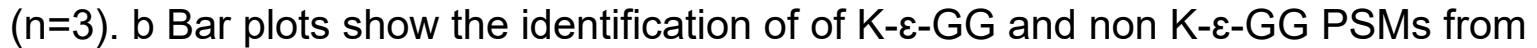
enrichments with on-antibody labeling with single channel TMT $(n=3)$. c Stacked bar plots show the percentage of fully labeled, partially labeled, unlabeled and sequences with no capacity for labeling. $d$ Venn diagram showing overlap of peptides identified with agarose and magnetic beads e Table showing all raw values, including enrichment specificity for previously described experiments. 
bioRxiv preprint doi: https://doi.org/10.1101/2021.04.28.441860; this version posted June 23, 2021. The copyright holder for this preprint (which was not certified by peer review) is the author/funder, who has granted bioRxiv a license to display the preprint in perpetuity. It is made available under aCC-BY-NC-ND 4.0 International license.

Automating UbiFast for Multiplexed Ubiquitin Enrichment

a

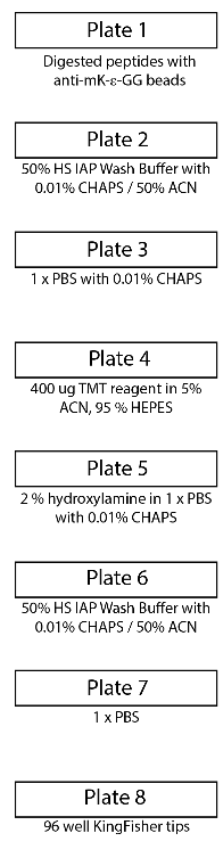

b Initial KingFisher setup

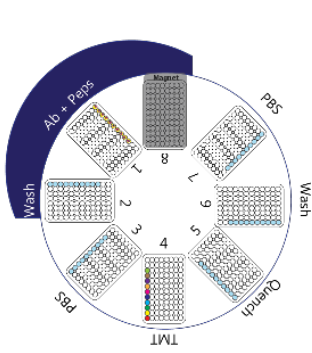

Step 4: On-antibody labeling with TMT

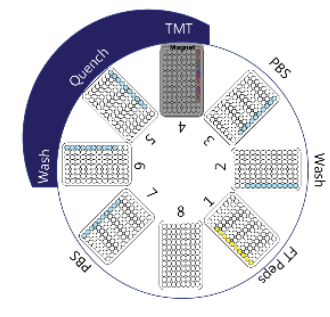

Step 1:

Collect beads from enrichment plate

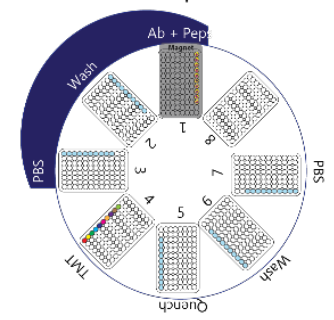

Step 5: Quench TMT reaction with hydroxylamine

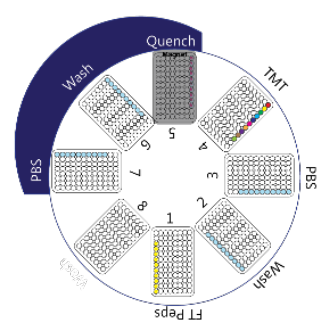

Step 2:

Wash beads with wash buffer and ACN

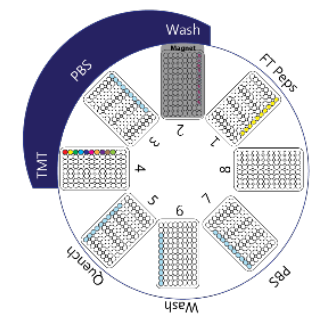

Step 6:

Wash beads with wash buffer and ACN

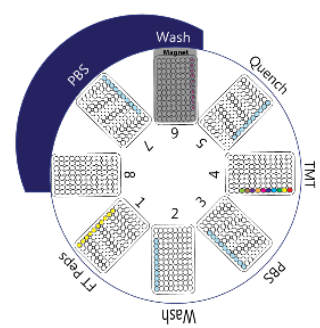

Step 3:

Wash beads with PBS

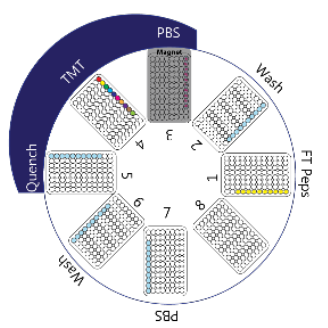

Step 7:

Release beads into PBS

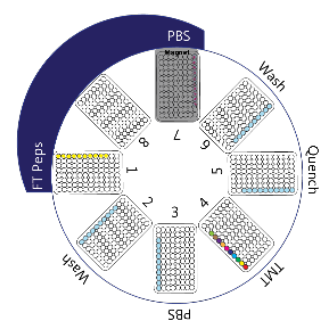

Supplemental Figure 2. Setup and processing steps for using the KingFisher Flex to automate ubiquitin enrichment. a Plate contents for each of the 896 -well KF plates. b Overview of each step in the automated ubiquitin enrichment process. 
Automating UbiFast for Multiplexed Ubiquitin Enrichment

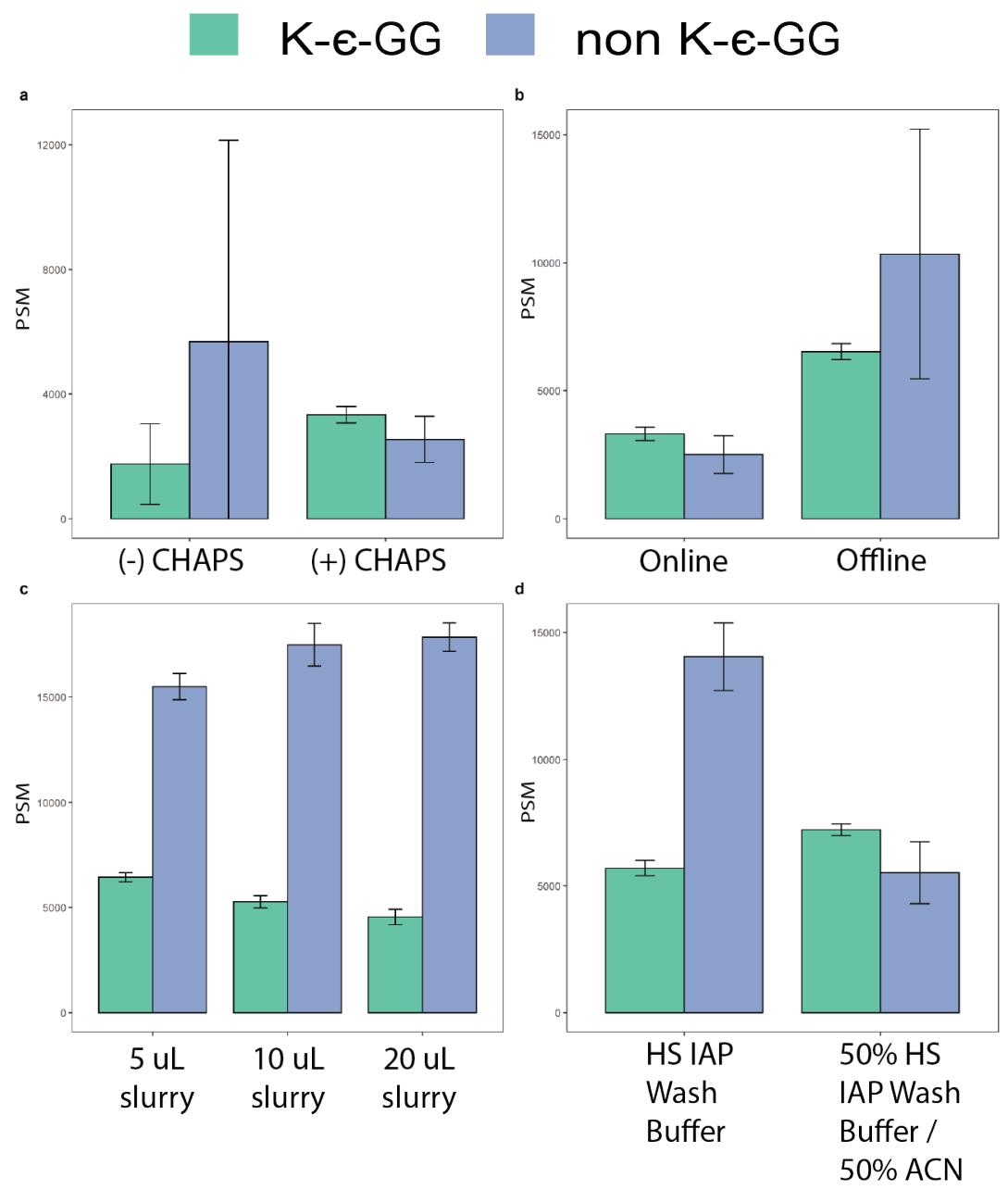

Supplemental Figure 3. Optimization of bead amounts and washing buffer for K-ع-GG enrichments using the KingFisher Flex instrument. a Bar plots showing identification of K-ع-GG PSMs when $0.01 \%$ CHAPS is either absent or present from buffers used on the KingFisher platform. b Bar plots showing identifications of K-ع-GG PSMs from enrichments where the binding of K- $\varepsilon$-GG peptides occurred for 1 hour on the KF robot

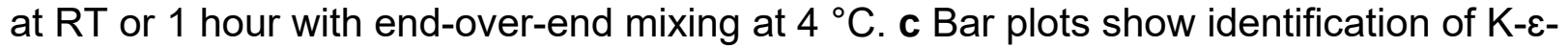
GG and non K-ع-GG PSM for enrichments using varying amounts of beads $(n=5)$. d Bar

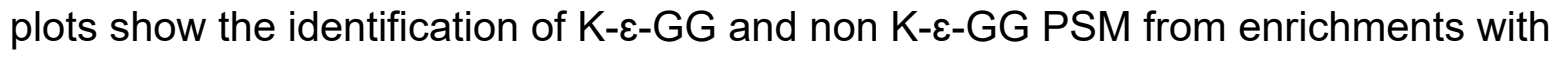
either HS IAP Wash Buffer or HS IAP Wash Buffer diluted 1:1 with ACN. 